together to form a large, ring-shaped molecule.

Valinomycin consists of three copies of a short depsipeptide (a tetradepsipeptide), which are joined together by the thioesterase domain of valinomycin synthase to form a $\mathrm{ring}^{4}$. Using the wild-type enzyme, HugueninDezot and colleagues first demonstrated that the thioesterase uses a 'retrograde' reaction mechanism to join the three tetradepsipeptides together. After the first tetradepsipeptide has been loaded onto the nucleophilic residue in the thioesterase domain, the second tetradepsipeptide arrives at the thioesterase active site, attached to the carrier domain. The first tetradepsipeptide is then transferred to, and reacts with, the second one, temporarily placing the resulting octadepsipeptide product on the carrier domain (rather than the second tetradepsipeptide being transferred to the first one on the thioesterase domain). The octadepsipeptide then migrates to the thioesterase domain, and the third tetradepsipeptide is brought to the active site to repeat the reaction cycle. This generates a dodecadepsipeptide that finally undergoes macrocyclization to form valinomycin.

The authors next incorporated DAP into the thioesterase domain to produce a stable acylenzyme intermediate that could be visualized using X-ray crystallography. The full structure of the dodecadepsipeptide could not be clearly visualized, but the residues that could be seen suggest that the active site of the thioesterase guides macrocyclization, partly by excluding water to minimize hydrolysis, and partly by directing the peptide's terminals towards each other for the reaction. The X-ray structure also shows that part of the thioesterase domain known as the lid region undergoes a substantial conformational change to orient the depsipeptide correctly for reaction.

When the serine or cysteine residue of an enzyme is replaced by DAP, the corresponding ester or thioester linkage in the acyl-enzyme intermediate is replaced by an amide linkage. It should be noted that this is not a perfect mimic of the natural linkage. The very feature of the amide that makes it resistant to hydrolysis - the fact that it behaves in part like a double bond - also constrains it to a planar geometry. Such a restriction does not apply for ester and thioester linkages. This is a minor issue, however, and does not diminish the value of visualizing the amide analogue of the natural acyl-enzyme intermediate.

Huguenin-Dezot and co-workers' method could be used to study various enzymatic systems, including enzymes of the ubiquitination pathway ${ }^{5}$ (which marks proteins for degradation), or any of the many types of hydrolase enzyme used in diverse bondcleaving processes ${ }^{6,7}$. Previous efforts to visualize the structures of acyl-enzyme intermediates have often used molecules that react irreversibly with the nucleophilic amino-acid residues in active sites ${ }^{8}$, to produce stable analogues of acyl-enzyme intermediates — but these might not mimic the ester (or thioester) linkage as closely as do Huguenin-Dezot and colleagues' amide analogues.

Furthermore, the new work could complement the use of ncAAs that bear reactive groups, which can be incorporated into large, multidomain enzymes to form covalent crosslinks between proteins or protein domains to trap functional states for structural studies. This has previously been done for polyketide synthase enzymes (which are closely related to NRPSs) at non-catalytic residues positioned at domain interfaces ${ }^{9}$. Huguenin-Dezot and co-workers' introduction of ncAA residues at positions that are responsible both for chemistry and for recognizing enzyme substrates or partner proteins, perhaps used in combination with chemical analogues of substrates and intermediates ${ }^{8}$, might uncover previously unknown structural features of biosynthetic enzymes.

Finally, DAP residues could potentially be used in a complementary method to existing approaches for profiling enzyme function ${ }^{10}$ : they could trap molecules from pools of metabolites in the active sites of uncharacterized hydrolases, and the bound molecule could be then identified using methods based on mass spectrometry to define the enzyme's preferred substrate. With such a wide variety of applications, Huguenin-Dezot and colleagues' method is a valuable addition to the protein chemist's toolbox that will advance the study of many important enzymes.

Andrew M. Gulick is in the Department of Structural Biology, Jacobs School of Medicine and Biomedical Sciences, University at Buffalo, Buffalo, New York 14203, USA.

e-mail:amgulick@buffalo.edu

1. Wang, L., Brock, A., Herberich, B. \& Schultz, P. G. Science 292, 498-500 (2001)

2. Huguenin-Dezot, N. et al. Nature 565, 112-117 (2019).

3. Payne, J. A., Schoppet, M., Hansen, M. H. \& Cryle, M. J. Mol. Biosyst. 13, 9-22 (2016).

4. Cheng, Y. Q. ChemBioChem. 7, 471-477 (2006).

5. Olsen, S. K., Capili, A. D., Lu, X., Tan, D. S. \& Lima, C. D. Nature 463, 906-912 (2010).

6. Bachovchin, D. A. \& Cravatt, B. F. Nature Rev. Drug Discov. 11, 52-68 (2012).

7. Lentz, C. S. et al. Nature Chem. Biol. 14, 609-617 (2018).

8. Gulick, A. M. \& Aldrich C. C. Nat. Prod. Rep. $\mathbf{3 5}$ 1156-1184 (2018).

9. Ye, Z. \& Williams, G. J. Biochemistry 53, 7494-7502 (2014).

10. Backus, K. M. et al. Nature 534, 570-574 (2016).

This article was published online on 12 December 2018.

\title{
INFLUENZA
}

\section{All for one and one for all to fight flu}

\begin{abstract}
Antibodies have been engineered to recognize diverse strains of influenza, including both the $A$ and $B$ types of virus that cause human epidemics. Are we moving closer to achieving 'universal' protection against all flu strains?
\end{abstract}

\section{GARY J. NABEL \& JOHN W. SHIVER}

$\mathrm{T}$ he First World War, one of the world's deadliest conflicts, claimed approximately 20 million lives. But in the year that it ended, an even deadlier calamity swept the globe. The 1918 influenza pandemic is estimated to have killed between 50 million and 100 million people ${ }^{1}$. Within months, a simple virus took a greater toll on human life than had the brutal four-year war. Although flu vaccines now save countless lives and have undoubtedly helped to avert other worldwide pandemics, their manufacture must vary annually to match the current circulating viral strains. Flu continues to pose a threat to human health, and there is a pressing need to develop countermeasures that can confer broad protection against the different flu strains. Moreover, vaccines are usually less effective at providing protection for children and for older individuals than they are for the rest of the population ${ }^{2}$.

Writing in Science, Laursen et al. ${ }^{3}$ report that they have engineered antibodies that protect against diverse flu viruses in mice, and that notably provide protection against most viral strains from the two major types of virus that cause human disease: influenza A and influenza B. Obtaining such broad protection has been a challenge because both influenza $A$ and influenza B are composed of diverse strains, and developing 'universal' protection has been an elusive goal. If the authors' approach could be adapted for effective use in humans, it might help to prevent or contain the spread of new and evolving flu infections worldwide.

During the 1918 pandemic, the cause of the disease was unknown. Had a vaccine been available, it would probably have limited the global catastrophe. However, developing an effective flu vaccine is not straightforward because flu viruses can mutate rapidly ${ }^{4}$. The high level of mutation results in continuous variation in two key viral proteins over time. One of these is haemagglutinin, which is located on the surface of the virus (Fig. 1) and 

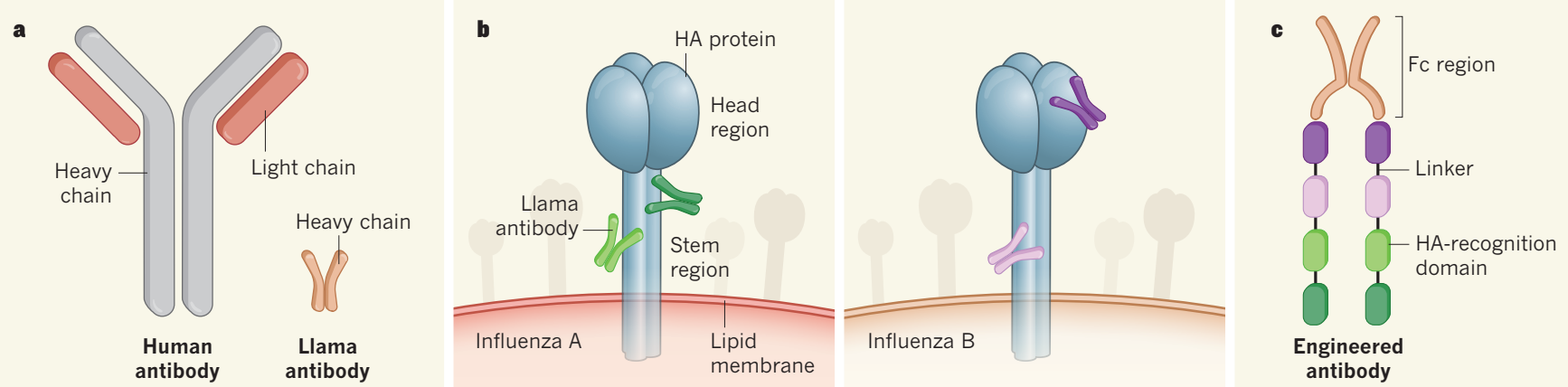

Figure 1 | Engineered antibodies target diverse strains of influenza virus. Laursen $e t$ al. ${ }^{3}$ report the development of antibodies that can provide broad protection against flu strains when tested in mice. a, The authors engineered antibodies based on antibodies from llamas (Lama glama), which are smaller than human antibodies. Llama antibodies, like human antibodies, contain regions known as heavy chains, but lack structures called light chains. b, The authors assessed llama antibodies that target the protein haemagglutinin (HA), which is found on the surface of the flu virus. In vitro analyses enabled the identification of antibodies that provide potent protection against the virus, and the authors isolated antibodies that targeted the two major groups of the virus: influenza $\mathrm{A}$ (green antibodies)and influenza B (purple antibodies). Structural analysis indicated whether the antibodies bound to the 'stem' or 'head' region of HA. c, The authors engineered antibodies containing such HA-recognition domains from llama antibodies, joined by linker regions (black). The antibodies also included an $\mathrm{Fc}$ region to aid interaction with immune cells. These antibodies provided protection against selected influenza A and B strains when tested in mice.

recognizes a molecule on host cells that serves as a receptor for viral attachment and entry.

Haemagglutinin also associates with a viral protein called neuraminidase. There are 18 distinct subtypes of haemagglutinin and 11 versions of neuraminidase. These two proteins form the basis for how flu strains are named. For example, the designation H1N1 indicates that a flu virus has haemagglutinin subtype 1 and neuraminidase subtype 1 .

A breakthrough in attempts to offer protection against diverse flu strains came with the identification of antibodies, termed broadly neutralizing antibodies, that can bind to a highly evolutionarily conserved and invariant structure in a region of haemagglutinin termed the stem ${ }^{5,6}$. Such antibodies battle the flu virus by binding haemagglutinin and inhibiting the virus's ability to enter cells. They can also boost an antiviral response, for example by engaging immune cells that promote the killing of virusinfected cells. However, these antibodies usually do not recognize all flu viruses. For instance, broadly neutralizing antibodies that recognize haemagglutinin of one major genetic subgroup of influenza A, group 1, typically do not react against the second group, group 2, and also do not recognize influenza $B$ (ref. 7).

To try to target influenza A and influenza B viruses, Laursen and colleagues had the idea of engineering an antibody by 'stitching' together influenza-recognition domains from different antibodies that bind to evolutionarily conserved regions of haemagglutinin, especially in the stem region of this protein. The authors vaccinated llamas (Lama glama) with a flu vaccine or haemagglutinin proteins, and used in vitro tests to identify resulting antibodies that had the greatest potency and breadth of neutralization against diverse flu viruses. They found that specific combinations of these antibodies could target nearly all of the flu-virus strains tested. Llama antibodies have a simpler structure and are smaller than human antibodies, and therefore aid an engineering approach that seeks to combine protein regions from more than one antibody.

By engineering antibodies in which several influenza-recognizing regions were connected by protein linkers, the authors were able to create antibodies that targeted multiple viruses. And fusing such structures to an antibody structure called the Fc region enabled such chimaeric proteins to interact with and activate immune cells.

When mice received either engineered antibodies or the gene encoding such an antibody - delivered by means of an adenoassociated virus (AAV) into cells of the nasal passage - they were protected against a flu virus that would usually have been lethal. The gene-delivery approach ensured production of the antibody for weeks to months, providing sustained protection without the need for multiple rounds of antibody injections over time.

Whether this approach could be used to prevent flu in humans is uncertain. Mice do not serve as optimal models for investigating human influenza because the receptor used by viral strains to infect mouse cells is a different version from that needed for cellular entry into human cells. In addition, the patterns of tissue infection and virus in the bloodstream often differ between mice and humans ${ }^{8}$. Protection in mice can involve a pathway that is mediated by a receptor protein called $\mathrm{F}_{\mathrm{C}} \gamma \mathrm{R}$-III on immune cells that recognizes antibodies bound to targets ${ }^{9}$, but whether this type of immune mechanism has relevance for humans is unknown. Antibodies that target the stem region of haemagglutinin have so far failed to alleviate symptoms in humans who are already infected, and the ability of these antibodies to prevent infection is being tested in clinical trials ${ }^{10}$.

Another worry regarding this approach in humans is whether an immune response might be triggered against the non-human antibodies. Although an engineered llama antibody has been approved for clinical use to treat a blood-clotting condition ${ }^{11}$, whether an immune response is generated against the anti-flu multi-domain antibodies will become clear only with clinical testing. Llama antibodies can be 'humanized' (engineered to closely resemble the related domains of human antibodies), yet the efficacy of such modifications would need to be evaluated in humans.

Also of concern is the use of AAV, because there are limitations to achieving sufficient and sustained levels of gene expression when using this virus in gene-therapy treatments ${ }^{12}$. Other safety and regulatory concerns regarding AAVs relate to their use to drive continuous gene expression, because this raises the possibility that complexes of human antibodies bound to the engineered antibodies might form over time. That said, certain groups, such as older people, might especially benefit from engineered antibodies, given the high mortality rates from flu in such individuals, and the fact that their immune responses tend to be less robust than those of younger adults.

The expression of engineered antibodies through gene-delivery approaches might offer a way of preventing or treating diverse types of infectious disease. Moreover, the outcomes of such treatments might help to confirm useful targets for the development of antiviral drugs or vaccines. For example, if broadly neutralizing antibodies that target the stem region of haemagglutinin can prevent flu infection in vivo in humans, it would encourage efforts to generate such antibodies by vaccination approaches. Antibodies targeting the stem region of haemagglutinin have previously been generated using structure-based approaches for vaccine design, and have shown promise in preclinical tests using animal models ${ }^{13-15}$.

Laursen and colleagues' approach of generating an antibody that can target more than one site is reminiscent of earlier work in which an antibody was developed ${ }^{16}$ from broadly neutralizing antibodies to target three independent sites on the HIV virus. Such antibodies can neutralize more than $99 \%$ of circulating HIV strains. This antibody blocked infection from viruses that were unaffected if single antibody 
components of the trispecific antibody were used. The era of multi-specific target engagement by engineered antibodies has begun, and might lead to new countermeasures to protect human health.

Gary J. Nabel is at Sanofi, Cambridge, Massachusetts 02139, USA. John W. Shiver is at Sanofi Pasteur, Swiftwater, Pennsylvania 18370, USA.

e-mail:gary.nabel@sanofi.com
. Morens, D. M. \& Fauci, A. S. J. Infect. Dis. 195, 1018-1028 (2007).

2. Paules, C. \& Subbarao, K. Lancet $390,697-708$ (2017).

3. Laursen, N. S. et al. Science 362, 598-602 (2018),

4. Taubenberger, J. K. \& Kash, J. C. Cell Host Microbe 7 , 440-451 (2010)

5. Ekiert, D. C et al. Science 324, 246-251 (2009)

6. Sui, J. et al. Nature Struct. Mol. Biol. 16, 265-273 (2009)

7. Corti, D. et al. Science 333, 850-856 (2011).

8. Margine, I. \& Krammer, F. Pathogens 3, 845-874 (2014).

9. Jegaskanda, S., Reading, P. C. \& Kent, S. J. J. Immunol. 193, 469-475 (2014).
10.Nachbagauer, R. \& Krammer, F. Clin. Microbiol. Infect. 23, 222-228 (2017).

11. Peyvandi, F. et al. N. Engl. J. Med. 374, 511-522 (2016)

12 Borsotti, C. \& Follenzi, A. Expert Rev. Clin Immunol.

14, 1013-1019 (2018)

13. Hai, R. et al. J. Virol. 86, 5774-5781 (2012).

14. Impagliazzo, A. et al. Science 349, 1301-1306 (2015)

15. Yassine, H. M. et al. Nature Med. 21, 1065-1070 (2015).

16.Xu, L. et al. Science 358, 85-90 (2017).

The authors declare competing financial interests. See go.nature.com/2zm2zhu for details.

This article was published online on 10 December 2018.

\section{Greenland's subglacial methane released}

suited to exporting methane. During winter, some meltwater from previous summers is stored in the inactive subglacial hydrological system $^{10}$. Lamarche-Gagnon et al. hypothesize that this winter storage allows meltwater to become enriched with methane through interaction with sediments in an oxygen-free environment.

In the spring, the subglacial hydrological system reactivates when it is flooded by the drainage of surface meltwater through crevasses and moulins (vertical conduits that allow meltwater to flow from the glacier surface to the bed beneath the ice sheet). This flooding causes an increase in ice motion ${ }^{11}$, and Lamarche-Gagnon and colleagues show that this flushes the methane-enriched, subglacial water to the ice margin. The authors observed multiple distinct flushing events after the activation of the subglacial hydrological system, suggesting that various types of meltwater pulse - drainage of lakes on the ice surface through moulins, rapid surface melting during warm spells, and upstream expansion of active subglacial water systems can liberate subglacial methane.

The observations indicate that methane concentrations tend to peak following sediment-rich proglacial discharge. The slight delay between peak methane export and proglacial discharge suggests that drainage of methane-rich water occurs just after the lateral flow of water that is associated with the greatest ice motion, and during times when differences in water pressure allow the drainage of normally isolated regions of the ice-sheet bed into enlarged subglacial channels ${ }^{12,13}$.

varied substantially in the past, and it has been hypothesized $^{2}$ that large reserves of methane can form and be trapped under ice sheets and glaciers when there is a favourable combination of carbon-rich sediments, high subglacial pressures, oxygen-poor conditions and low temperatures. Rapid release of this methane during glacial retreat might trigger rapid warming ${ }^{3}$, but whether large-scale release of such glacial methane could occur in the future is disputed ${ }^{4}$.

Field observations provide equivocal evidence for whether subglacial sediments act as a source or sink of methane. Ice-core drilling operations in West Antarctica detected methane-producing microbes in proglacial and subglacial sediments ${ }^{5}$, but analyses of Antarctic subglacial lake sediments ${ }^{6}$ and proglacial

sediments ${ }^{7}$ indicate that bacterial oxidation consumes almost all the methane produced preventing its release to the atmosphere. This subglacial hy

Lamarche-Gagnon et al. show that subthe atmosphere within a well-characterized subglacial catchment (an area within which subglacial water collects and drains out of a common outlet) in Greenland. Subglacia merefore act as a local sou of methane, corroborating the results of other recent studies of subglacial methane $e^{8,9}$. Lamarche-Gagnon et al. go further by demonstrating that the continuous flux of methane from the Greenland subglacial environment varies with the efficiency of subglacial meltwater drainage.

Near the margins of the Greenland Ice Sheet, the glacial hydrological system seems to be well

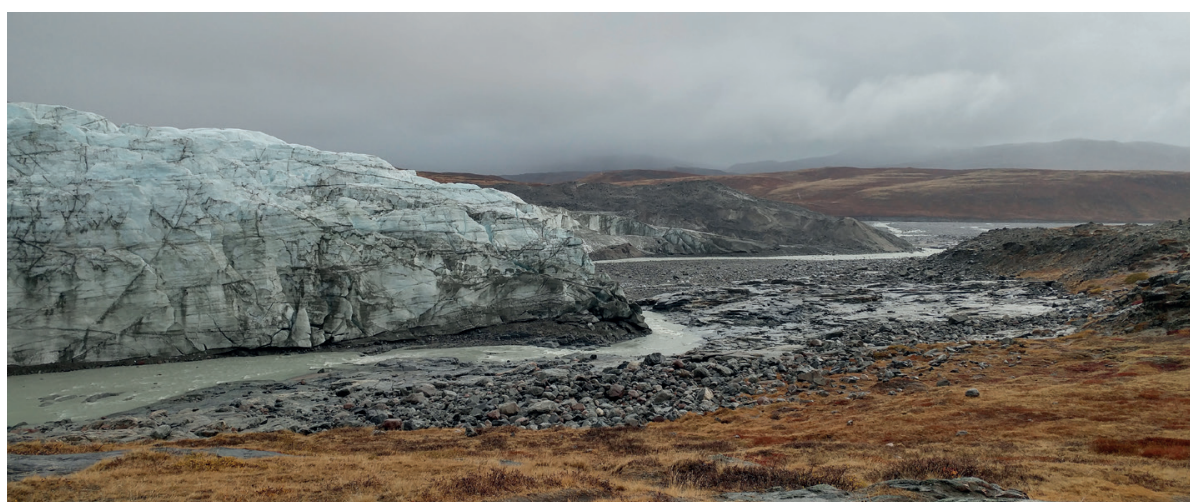

Figure 1 | Sediment-rich meltwater released from Russell Glacier, Greenland. Lamarche-Gagnon et al. ${ }^{1}$ report that such discharge contains concentrations of methane equal to those of many terrestrial rivers. 